\title{
A COMPARISON OF THE NEUROPROTECTIVE EFFICACY OF PHARMACOLOGICAL PRETREATMENT AND ANTIDOTAL TREATMENT IN SOMAN-POISONED RATS
}

\author{
Jiř́ Kassa', Gabriela Krejčová ${ }^{1}$, Ivan Samnaliev²
}

Purkyně Military Medical Academy in Hradec Králové: Department of Toxicology ${ }^{1}$; Military Medical Academy in Sofia, Bulgaria: Department of Experimental Toxicology 2

Summary: 1. To study the influence of pharmacological pretreatment (PANPAL or pyridostigmine combined with biperiden) and antidotal treatment (the oxime HI-6 plus atropine) on soman-induced neurotoxicity, male albino rats were poisoned with a lethal dose of soman (54 (g/kg i.m.; 100\% of $\mathrm{LD}_{50}$ value) and observed at 24 hours and 7 days following soman challenge. The neurotoxicity of soman was evaluated using a Functional observational battery and an automatic measurement of motor activity. 2. Pharmacological pretreatment as well as antidotal treatment were able to eliminate some of soman-induced neurotoxic effects observed at 24 hours following soman poisoning. The combination of pharmacological pretreatment (PANPAL or pyridostigmine combined with biperiden) and antidotal treatment was found to be more effective in the elimination of soman-induced neurotoxicity in rats at 24 hours following soman challenge in comparison with the administration of pharmacological pretreatment or antidotal treatment alone. To compare both pharmacological pretreatments, the combination of pyridostigmine with biperiden seems to be more efficacious to eliminate soman-induced signs of neurotoxicity than PANPAL. 3. At 7 days following soman poisoning, the combination of pharmacological pretreatment involving pyridostigmine and biperiden with antidotal treatment was only able to completely eliminate somaninduced neurotoxic signs. 4. Thus, our findings confirm that the combination of pharmacological pretreatment and antidotal treatment is able not only to protect the experimental animals from the lethal effects of soman but also to eliminate most soman-induced signs of neurotoxicity in poisoned rats. The pharmacological pretreatment containing pyridostigmine and biperiden appears to be more efficacious to eliminate soman-induced neurotoxic sings than PANPAL.

Key words: Soman; Functional observational battery; Motor activity; PANPAL, Pyridostigmine; Biperiden; Atropine; HI-6; Rats

\section{Introduction}

Despite of the entry into force in April 1997 of the Chemical Weapons Convention forbidding the production, storage and use of chemical warfare agents, the world has seen a rapid proliferation of such agents, especially nerve agents. Therefore, inactivation of extremely toxic organophosphorus compounds (nerve agents) has become a subject of major importance. The international control of their proliferation is thwarted by the ease of their synthesis and by similarity between their chemical precursors and widely used pest-control agents. Their harmful effect is related to their potency to irreversibly inhibit mammalian acetylcholinesterase (AChE, EC 3.1.1.7), the enzyme responsible for the regulation of neurotransmitter acetylcholine (ACh) concentration at cholinergic synapses (23). The inhibition of $\mathrm{AChE}$ induces a major increase in ACh level in the cholinergic nervous system producing muscle fasciculations, respiratory distress and epileptic fits leading to the generalized seizures. In surviving animals, the seizures lead to severe incapacitation and to irreversible brain damage with lesions especially in hippocampus, piriform cortex $(17,21)$ and other cortical structures (13).

The current antidotal treatment of nerve agent-induced acute poisoning usually consists of anticholinergic drugs to antagonize the effects of ACh excess at cholinergic receptor sites and oximes to reactivate nerve agent-inhibited AChE $(4,7,20)$. Unfortunately, some organophosphates were found to be resistant to standard antidotal treatment. One of the most resistant organophosphorus compound is soman (pinacolyl methylphosphonofluoridate). Its deleterious effects are extraordinarily difficult to counteract because of rapid aging of soman-inhibited AChE (2).

The relatively unsatisfactory treatment available for acute nerve agent poisoning has prompted studies of pretreatment possibilities that allow survival and increase re- 
sistance of organisms exposed to nerve agents. Currently used method of protection against nerve agent poisoning is the use of pyridostigmine bromide, a reversible carbamate AChE inhibitor (1). Prophylactic effect of pyridostigmine can result from its reversible inhibition of AChE. It binds a small fraction of $\mathrm{AChE}$ in the periphery and reversibly shields it from irreversible inhibition by the nerve agents (3). However, pyridostigmine-induced increase in the level of ACh can itself cause symptoms of poisoning. Therefore, it would be useful to counteract the effects of the accumulated ACh using anticholinergic drugs. In addition, the combination of pyridostigmine with anticholinergic drugs allows to increase the dose of pyridostigmine because the anticholinergic drugs are able to counteract side cholinergic effects of pyridostigmine $(8,10)$. One of these mixtures, pyridostigmine in combination with benactyzine (BNZ) and trihexyphenidyle (THP), designated PANPAL, has been developed in the Czech Republic and introduced to the Czech Army (22). Another mixture, pyridostigmine in combination with biperiden, has been developed in Bulgaria (18).

The aim of this study was to compare the neuroprotective effects of both pharmacological pretreatment mixtures with or without antidotal treatment consisting of HI-6 and atropine in soman-poisoned rats. The soman-induced neurotoxic signs were determined using a Functional observational battery, a non-invasive neurological examination containing measurements of sensory, motor and autonomic nervous functions.

\section{Methods}

Animals used in our experiments were male albino Wistar rats weighing 180-220 g purchased from Konárovice (Czech Republic). They were kept in an air-conditioned room and allowed to access to standard food and tap water ad libitum. The rats were divided into groups of eight animals $(n=8)$. Handling of the experimental animals was done under the supervision of the Ethics Committee of the Medical Faculty of Charles University and Purkyne Military Medical Academy in Hradec Králové (Czech Republic).

Soman of $98.5 \%$ purity was obtained from Zemianské Kostolany (Slovak Republic). Its purity was assayed by acidimetric titration. The oxime HI-6 was synthesised at the Department of Toxicology of the Military Medical Academy and was $98 \%$ pure. Its purity was analysed using HPLC. All other chemicals and drugs of analytical grade were obtained commercially and used without further purification.

Pyridostigmine $(0.75 \mathrm{mg} / \mathrm{kg}$ of body weight $)$ in combination with BNZ (16 mg/kg of body weight) and THP (6.3 $\mathrm{mg} / \mathrm{kg}$ of body weight) or pyridostigmine $(0.75 \mathrm{mg} / \mathrm{kg}$ of body weight) in combination with bideriden $(2.5 \mathrm{mg} / \mathrm{kg}$ of body weight) was administered intramuscularly (i.m.) as solution in distilled water $(0.1 \mathrm{ml} / 100 \mathrm{~g}$ of body weight $) 30$ min before i.m. soman challenge at a lethal dose $(54 \mu \mathrm{g} / \mathrm{kg}$ b.w. $-\mathrm{LD}_{50}$ ). Antidotal treatment (HI-6 in combination with atropine) was carried out by i.m. injection $1 \mathrm{~min}$ following soman administration. The doses of HI-6 (15.6 mg/kg of body weight) and anticholinergic drug atropine $(10 \mathrm{mg} / \mathrm{kg}$ of body weight) correspond to human-relevant doses ( $2 \%$ of their $\mathrm{LD}_{50}$ ) (3). The neurotoxicity of soman was monitored using the Functional observational battery (FOB) at 24 hours and 7 days following soman poisoning. The evaluated markers of soman-induced neurotoxicity in experimental animals were compared to the parameters obtained from control rats, administered with saline instead of soman and antidotes at the same volume $(0.1 \mathrm{ml} / 100 \mathrm{~g} \mathrm{~b}$. w. $)$.

The FOB consists of 40 measures of sensory, motor and autonomic functions (Tab. 1) $(5,14,15,16)$. First measurements were made while the animal was in the home cage. The observer evaluated each animal posture, palpebral closure, and presence or absence of convulsions. If convulsions were present, they were further categorized. Following observations in the home cage, the animal was removed and briefly held in the hand. Ease of removal and handling, skin and fur abnormalities, lacrimation, salivation and nose secretion were recorded.

Then, the rat was placed on a flat surface, which served as the open field covered with a clean absorbent pad. A timer was started for three minutes during which time the frequency of rearing responses was noted. At the same time, gait characteristics were noted and ranked, and arousal, tremor, convulsions and abnormal postures were evaluated. At the end of the time period ( $3 \mathrm{~min}$ ), the number of faecal boluses and urine pools on the absorbent pad were recorded. Reflex testing followed next and consisted of recording each rat's responses to the frontal approach of a blunt object such as a pencil, a touch of an object to the posterior flank, and an auditory click stimulus. Reactivity to a pinch on the tail and the ability of the pupil to constrict in response to light were also assessed. These examinations were followed by a test for the righting reflex, then by measurements of forelimb and hindlimb grip strength, rectal temperature and finally landing foot splay. The entire battery of tests required approximately from six to eight minutes per one rat.

Motor activity data were collected shortly after FOB testing, using an apparatus for testing a spontaneous motor activity of laboratory animals (constructed in Purkyne Military Medical Academy, Hradec Králové, Czech Republic). The animals were placed into the measuring cage for a short time (10 minutes) and their movements (total, horizontal and vertical activity) were recorded.

Data collected with the FOB and motor activity assessment include categorical, ordinal and continuous values. Statistical analyses were performed on a PC with a special interactive programme NTX (5). The categorical and ordinal values were formulated as contingency tables and judged consecutively by Chi-squared test of homogeneity, Concordance-Discordance test and Kruskal-Wallis test respectively. The continual data were assessed by successive statistical tests: CI for Delta, Bartlett test for Equality of Variance, Williams test and Test for Distribution Function. The differences were considered significant when $\mathrm{p}<0.05$. 
Tab. 1: Functional Observational Battery (FOB).

\begin{tabular}{|c|c|c|c|c|c|c|c|c|c|c|}
\hline MARKER & \multicolumn{10}{|c|}{ Scored values only } \\
\hline & -2 & -1 & 0 & 1 & 2 & 3 & 4 & 5 & 6 & 7 \\
\hline POSTURE & & & & $\begin{array}{l}\text { sitting or } \\
\text { standing }\end{array}$ & rearing & asleep & flattened & $\begin{array}{l}\text { lying on } \\
\text { side }\end{array}$ & $\begin{array}{l}\text { crouched } \\
\text { over }\end{array}$ & $\begin{array}{c}\text { head } \\
\text { bobbing }\end{array}$ \\
\hline $\begin{array}{l}\text { CATCH } \\
\text { DIFFICULTY }\end{array}$ & & & & passive & normal & $\begin{array}{l}\text { elevated } \\
\text { activity }\end{array}$ & flight & escape & $\begin{array}{l}\text { aggrres- } \\
\text { sion }\end{array}$ & \\
\hline $\begin{array}{l}\text { EASE OF } \\
\text { HANDLING }\end{array}$ & & & & passive & normal & $\begin{array}{l}\text { modera- } \\
\text { tely } \\
\text { difficult }\end{array}$ & difficult & & & \\
\hline $\begin{array}{l}\text { MUSCULAR } \\
\text { TONUS }\end{array}$ & atonia & $\begin{array}{l}\text { hypo- } \\
\text { tonia }\end{array}$ & normal & $\begin{array}{c}\text { hyper- } \\
\text { tonia }\end{array}$ & rigidity & $\begin{array}{c}\text { fascicula- } \\
\text { tions } \\
\end{array}$ & & & & \\
\hline LACRIMATION & & & none & slight & severe & crusta & $\begin{array}{l}\text { coloured } \\
\text { crusta }\end{array}$ & & & \\
\hline $\begin{array}{l}\text { PALPEBRAL } \\
\text { CLOSURE }\end{array}$ & & & & open & $\begin{array}{l}\text { slightly } \\
\text { drooping }\end{array}$ & half-way & $\begin{array}{c}\text { comple- } \\
\text { tely } \\
\text { shut }\end{array}$ & ptosis & & \\
\hline $\begin{array}{l}\text { ENDO-EXO- } \\
\text { PHTHALMUS }\end{array}$ & & endo & normal & exo & & & & & & \\
\hline $\begin{array}{l}\text { FUR ABNORMA- } \\
\text { LITIES }\end{array}$ & & & normal & coloured & tousled & $\begin{array}{l}\text { color. } \\
\text { +tousl. }\end{array}$ & blaze & injury & $\begin{array}{c}\text { other } \\
\text { changes }\end{array}$ & $\begin{array}{c}\text { pilo- } \\
\text { erection }\end{array}$ \\
\hline $\begin{array}{l}\text { SKIN ABNORMA- } \\
\text { LITIES }\end{array}$ & & & normal & pale & erythema & cyanosis & $\begin{array}{c}\text { pig- } \\
\text { mented }\end{array}$ & cold & injury & \\
\hline SALIVATION & & & none & sllight & severe & & & & & \\
\hline NOSE SECRETION & & & none & slight & severe & coloured & & & & \\
\hline $\begin{array}{l}\text { CLONIC } \\
\text { MOVEMENTS }\end{array}$ & & & normal & repetitive & $\begin{array}{c}\text { non- } \\
\text { rhythmic }\end{array}$ & $\begin{array}{c}\text { mild } \\
\text { tremors }\end{array}$ & $\begin{array}{l}\text { severe } \\
\text { tremors }\end{array}$ & $\begin{array}{c}\text { myoclonic } \\
\text { jerks }\end{array}$ & clonic & $\begin{array}{c}\text { wet dog } \\
\text { shakes }\end{array}$ \\
\hline $\begin{array}{l}\text { TONIC } \\
\text { MOVEMENTS }\end{array}$ & & & normal & $\begin{array}{l}\text { contrac- } \\
\text { tion of } \\
\text { extensors }\end{array}$ & $\begin{array}{l}\text { opistho- } \\
\text { tonus }\end{array}$ & $\begin{array}{l}\text { empros- } \\
\text { thotonus }\end{array}$ & $\begin{array}{l}\text { explosive } \\
\text { jumps }\end{array}$ & $\begin{array}{c}\text { tonic } \\
\text { con- } \\
\text { vulsions } \\
\end{array}$ & & \\
\hline GAIT & & & normal & ataxia & $\begin{array}{c}\text { overcom- } \\
\text { pensation } \\
\text { of hind- } \\
\text { limbs } \\
\text { move- } \\
\text { ments }\end{array}$ & $\begin{array}{c}\text { feet } \\
\text { point } \\
\text { outwards } \\
\text { from } \\
\text { body }\end{array}$ & $\begin{array}{l}\text { forelimbs } \\
\text { are } \\
\text { extended }\end{array}$ & $\begin{array}{c}\text { walks } \\
\text { on tiptoes }\end{array}$ & $\begin{array}{c}\text { hunched } \\
\text { body }\end{array}$ & $\begin{array}{c}\text { body is } \\
\text { flattened } \\
\text { against } \\
\text { surface }\end{array}$ \\
\hline GAIT SCORE & & & & normal & $\begin{array}{l}\text { slightly } \\
\text { impaired }\end{array}$ & $\begin{array}{l}\text { somewhat } \\
\text { impaired }\end{array}$ & $\begin{array}{l}\text { totally } \\
\text { impaired }\end{array}$ & & & \\
\hline $\begin{array}{l}\text { MOBILITY } \\
\text { SCORE }\end{array}$ & & & & normal & $\begin{array}{l}\text { slightly } \\
\text { impaired }\end{array}$ & $\begin{array}{l}\text { somewhat } \\
\text { impaired }\end{array}$ & $\begin{array}{l}\text { totally } \\
\text { impaired }\end{array}$ & & & \\
\hline $\begin{array}{l}\text { AROUSAL (level of } \\
\text { unprovoked activity) }\end{array}$ & & & & very low & sporadic & reduced & normal & enhanced & permanent & \\
\hline TENSION & & & none & $\begin{array}{c}\text { partial } \\
\text { (ears) }\end{array}$ & stupor & & & & & \\
\hline STEREOTYPY & & & none & $\begin{array}{c}\text { head } \\
\text { weaving }\end{array}$ & $\begin{array}{c}\text { body } \\
\text { weaving }\end{array}$ & grooming & circling & others & & \\
\hline $\begin{array}{l}\text { BIZARRE } \\
\text { BEHAVIOR }\end{array}$ & & & none & head & body & $\begin{array}{c}\text { self- } \\
\text { mutila } \\
\text { tion }\end{array}$ & $\begin{array}{c}\text { abnormal } \\
\text { move- } \\
\text { ments }\end{array}$ & others & & \\
\hline $\begin{array}{l}\text { APPROACH } \\
\text { RESPONSE }\end{array}$ & & & & $\begin{array}{c}\text { no } \\
\text { reaction }\end{array}$ & normal & freeze & $\begin{array}{l}\text { energetic } \\
\text { reaction }\end{array}$ & $\begin{array}{l}\text { exagge- } \\
\text { rated } \\
\text { reaction }\end{array}$ & & \\
\hline $\begin{array}{l}\text { TOUCH } \\
\text { RESPONSE }\end{array}$ & & & & $\begin{array}{c}\text { no } \\
\text { reaction }\end{array}$ & normal & freeze & $\begin{array}{l}\text { energetic } \\
\text { reaction }\end{array}$ & $\begin{array}{l}\text { exagge- } \\
\text { rated } \\
\text { reaction }\end{array}$ & & \\
\hline $\begin{array}{l}\text { CLICK } \\
\text { RESPONSE }\end{array}$ & & & & $\begin{array}{c}\text { no } \\
\text { reaction }\end{array}$ & normal & freeze & $\begin{array}{l}\text { energetic } \\
\text { reaction }\end{array}$ & $\begin{array}{l}\text { exagge- } \\
\text { rated } \\
\text { reaction }\end{array}$ & & \\
\hline $\begin{array}{l}\text { TAIL - PINCH } \\
\text { RESPONSE }\end{array}$ & & & & $\begin{array}{c}\text { no } \\
\text { reaction }\end{array}$ & normal & freeze & $\begin{array}{l}\text { energetic } \\
\text { reaction }\end{array}$ & $\begin{array}{l}\text { exagge- } \\
\text { rated } \\
\text { reaction }\end{array}$ & & \\
\hline PUPIL SIZE & $\begin{array}{c}\text { miosis } \\
\text { consider- } \\
\text { able }\end{array}$ & $\begin{array}{l}\text { miosis } \\
\text { slight }\end{array}$ & normal & $\begin{array}{l}\text { mydriasis } \\
\text { slight }\end{array}$ & $\begin{array}{l}\text { mydriasis } \\
\text { slight }\end{array}$ & $\begin{array}{c}\text { mydriasis } \\
\text { consider- } \\
\text { able }\end{array}$ & & & & \\
\hline PUPIL RESPONSE & & & $\begin{array}{c}\text { no } \\
\text { reaction }\end{array}$ & $\begin{array}{l}\text { normal } \\
\text { reaction }\end{array}$ & & & & & & \\
\hline $\begin{array}{l}\text { RIGHTING } \\
\text { REFLEX }\end{array}$ & & & & normal & \begin{tabular}{|c|} 
slightly \\
uncoordin.
\end{tabular} & $\begin{array}{l}\text { lands on } \\
\text { side }\end{array}$ & $\begin{array}{l}\text { lands on } \\
\text { back }\end{array}$ & $\begin{array}{c}\text { rise from } \\
\text { back spon- } \\
\text { taneous }\end{array}$ & $\begin{array}{l}\text { rise from } \\
\text { back with } \\
\text { stimulus }\end{array}$ & $\begin{array}{c}\text { no } \\
\text { reaction }\end{array}$ \\
\hline
\end{tabular}


F Tab. 2: The values of soman-induced neurotoxic markers $(\mathrm{x} \pm \mathrm{s})$ measured at $24 \mathrm{~h}$ following soman challenge by FOB $(1-32-$ scored values, No $33-41-$ values in absolute units). Statistical significance (comparison to control values) $-{ }^{*} p<0.05,{ }^{* *} p<0.01,{ }^{* * *} p<0.001$.

\begin{tabular}{|c|c|c|c|c|c|c|c|c|c|c|c|c|c|c|c|}
\hline & 24 hours & Cont & & Soma & & $\begin{array}{l}\text { Panpa } \\
\text { Soma }\end{array}$ & & $\begin{array}{r}\text { Pyridostig } 1 \\
\text { Biperid } \\
+ \text { Som }\end{array}$ & $\begin{array}{l}\operatorname{mine}+ \\
\text { len } \\
\text { an }\end{array}$ & $\begin{array}{r}\text { Soman }+ \\
\text { HI- } 6\end{array}$ & & $\begin{array}{r}\text { Panpa } \\
\text { Soman + } \\
\text { HI- } 6\end{array}$ & & $\begin{array}{r}\text { Pyridosti } \\
+ \text { Biperi } \\
+ \text { Somar } \\
+ \text { HI- }\end{array}$ & $\begin{array}{l}\text { gmine } \\
\text { iden } \\
1+\mathrm{A} \\
6\end{array}$ \\
\hline No & Marker & $\overline{\mathrm{x}}$ & $\pm \mathrm{s}$ & $\overline{\mathrm{x}}$ & $\pm \mathrm{s}$ & $\overline{\mathrm{x}}$ & $\pm \mathrm{s}$ & $\overline{\mathrm{x}}$ & $\pm \mathrm{s}$ & $\overline{\mathrm{x}}$ & $\pm \mathrm{S}$ & $\overline{\mathrm{x}}$ & $\pm \mathrm{s}$ & $\bar{x}$ & $\pm \mathrm{S}$ \\
\hline 1 & posture & 1.13 & 0.35 & $3,88 * * *$ & 1.25 & 1.13 & 0.35 & 1.25 & 0.71 & 1.50 & 0.93 & 1.38 & 0.74 & 1.00 & 0.00 \\
\hline 2 & catch difficulty & 2.00 & 0.00 & $1,75^{* * *}$ & 1.75 & $1,25 * * *$ & 0.46 & $1,25 * * *$ & 0.46 & $2,63 * *$ & 0.92 & 2.00 & 0.00 & 2.13 & 0.35 \\
\hline 3 & ease of handling & 2.00 & 0.00 & $1,25^{* * *}$ & 0.46 & $1,25 * * *$ & 0.46 & $1,25^{* * *}$ & 0.46 & 1.86 & 0.38 & 2.00 & 0.00 & 2.00 & 0.00 \\
\hline 4 & muscular tonus & 0.00 & 0.00 & $-1,38^{* * * *}$ & 0.74 & $-0,63^{* *}$ & 0.52 & -0.13 & 0.35 & $-0,71^{* * *}$ & 0.49 & $-1,00^{* * * *}$ & 0.00 & -0.25 & 0.71 \\
\hline 5 & lacrimation & 0.00 & 0.00 & $2,13 * * *$ & 1.25 & $0,5^{*}$ & 0.53 & $0,75^{* * *}$ & 0.46 & $0,88^{* * *}$ & 0.35 & 0.00 & 0.00 & 0.00 & 0.00 \\
\hline 6 & palpebral closure & 1.00 & 0.00 & $2,25^{*}$ & 1.39 & 1.00 & 0.00 & 1.00 & 0.00 & 1.00 & 0.00 & 1.00 & 0.00 & 1.00 & 0.00 \\
\hline 7 & endo-exophthalmus & 0.00 & 0.00 & 0.00 & 0.76 & 0.00 & 0.00 & 0.00 & 0.00 & $0,57^{*}$ & 0.53 & 0.00 & 0.00 & 0.00 & 0.00 \\
\hline 8 & fur abnormalities & 0.00 & 0.00 & $4,38^{* * *}$ & 3.62 & 0.00 & 0.00 & 0.00 & 0.00 & 0.00 & 0.00 & 0.00 & 0.00 & 0.13 & 0.35 \\
\hline 9 & skin abnormalities & 0.00 & 0.00 & $1,75 * *$ & 0.00 & 0.00 & 0.00 & 0.75 & 1.04 & $2,00 * * *$ & 0.00 & 0.00 & 0.00 & 0.75 & 1.04 \\
\hline 10 & salivation & 0.00 & 0.00 & 0.38 & 0.74 & 0.00 & 0.00 & 0.00 & 0.00 & $0,71^{* * *}$ & 0.49 & 0.00 & 0.00 & 0.00 & 0.00 \\
\hline 11 & nose secretion & 0.00 & 0.00 & $1,38^{*}$ & 1.41 & $2,13 * * *$ & 1.25 & $0,75^{* * *}$ & 0.46 & $1,14^{* * *}$ & 0.38 & 0.00 & 0.00 & 0.38 & 1.06 \\
\hline 12 & rearing & 16.38 & 4.41 & $4,38 * * *$ & 6.12 & $7,38^{*}$ & 7.60 & 9.63 & 8.38 & 8.14 & 9.87 & $2,63^{* * *}$ & 3.02 & $5,00 * * *$ & 3.70 \\
\hline 13 & urination & 0.88 & 1.64 & 0.00 & 0.00 & 0.38 & 1.06 & 1.75 & 4.56 & 0.38 & 0.74 & 0.25 & 0.71 & 1.75 & 4.95 \\
\hline 14 & defecation & 0.63 & 1.41 & 0.13 & 0.35 & 0.38 & 0.52 & 0.25 & 0.71 & 0.00 & 0.00 & 0.00 & 0.00 & 0.25 & 0.71 \\
\hline 15 & clonic movements & 0.00 & 0.00 & $1,88^{*}$ & 2.36 & 1.00 & 2.14 & $1,75^{* *}$ & 1.49 & $2,29 * * *$ & 1.11 & 1.13 & 1.55 & $1,38^{*}$ & 1.60 \\
\hline 16 & tremor & 0.00 & 0.00 & 0.29 & 0.71 & 0.00 & 0.00 & $1,43^{*}$ & 1.39 & $2,00 * * *$ & 1.36 & 1.14 & 1.41 & 0.71 & 1.19 \\
\hline 17 & tonic movements & 0.00 & 0.00 & 0.50 & 1.07 & 0.00 & 0.00 & 0.00 & 0.00 & 0.14 & 0.38 & 0.00 & 0.00 & 0.00 & 0.00 \\
\hline 18 & gait & 0.50 & 0.93 & $5,38^{* * * *}$ & 2.00 & $3,38 * * *$ & 1.92 & 0.50 & 0.76 & 1.00 & 1.00 & $1,75^{*}$ & 0.71 & 0.63 & 1.77 \\
\hline 19 & ataxia & 0.00 & 0.00 & $1,13^{* * *}$ & 0.83 & 0.38 & 0.52 & 0.25 & 0.46 & 0.43 & 0.79 & 0.00 & 0.00 & 0.00 & 0.00 \\
\hline 20 & gait score & 1.25 & 0.46 & $2,88^{*}$ & 0.99 & $2,25^{*}$ & 0.71 & 1.63 & 0.52 & $2,00^{* * *}$ & 0.00 & $1,88^{*}$ & 0.35 & 1.63 & 0.52 \\
\hline 21 & mobility score & 1.00 & 0.00 & $2,50^{*}$ & 1.41 & 1.50 & 0.53 & 1.13 & 0.35 & 1.29 & 0.49 & 1.00 & 0.00 & 1.00 & 0.00 \\
\hline 22 & arousal & 4.25 & 0.46 & $2,75^{*}$ & 1.49 & $3,25^{*}$ & 0.89 & 3.88 & 0.99 & 4.00 & 1.00 & 3.88 & 0.35 & $3,50^{*}$ & 0.53 \\
\hline 23 & tension & 0.00 & 0.00 & 0.00 & 0.00 & 0.00 & 0.00 & 0.00 & 0.00 & 0.00 & 0.00 & 0.00 & 0.00 & 0.38 & 0.74 \\
\hline 24 & stereotypy & 0.00 & 0.00 & 0.00 & 0.00 & 0.13 & 0.35 & 0.50 & 1.41 & 0.50 & 1.41 & 0.00 & 0.00 & 0.00 & 0.00 \\
\hline 25 & bizzare behavior & 0.00 & 0.00 & 0.00 & 0.00 & 0.00 & 0.00 & 0.00 & 0.00 & 0.00 & 0.00 & 0.00 & 0.00 & 0.00 & 0.00 \\
\hline 26 & approach response & 1.88 & 0.35 & 1.50 & 0.53 & 2.38 & 0.74 & 1.88 & 0.35 & 2.00 & 0.00 & 2.00 & 0.00 & 1.75 & 0.46 \\
\hline 27 & touch response & 1.88 & 0.35 & 1.50 & 0.53 & $2,13 * *$ & 0.99 & 1.75 & 1.04 & 2.00 & 0.00 & 2.13 & 0.35 & 1.50 & 0.53 \\
\hline 28 & click response & 2.13 & 0.35 & $1,38^{*}$ & 0.52 & 2.00 & 0.00 & 2.25 & 0.71 & 2.43 & 0.53 & 2.00 & 0.00 & 2.00 & 0.00 \\
\hline 29 & tail-pinch response & 2.00 & 0.00 & 1.88 & 0.35 & 1.75 & 0.46 & 1.63 & 0.52 & 2.00 & 0.00 & 2.00 & 0.00 & 1.63 & 0.52 \\
\hline 30 & pupil size & 0.00 & 0.00 & $-1,00^{*}$ & 1.07 & 0.00 & 0.00 & 0.00 & 0.00 & 0.00 & 0.00 & 0.00 & 0.00 & -0.13 & 0.35 \\
\hline 31 & pupil response & 1.00 & 0.00 & $0,5^{*}$ & 0.53 & 1.00 & 0.00 & 1.00 & 0.00 & 1.00 & 0.00 & 1.00 & 0.00 & 1.00 & 0.00 \\
\hline 32 & righting reflex & 1.00 & 0.00 & $3,00^{*}$ & 1.77 & 1.00 & 0.00 & 1.00 & 0.00 & 1.00 & 0.00 & 1.00 & 0.00 & 1.00 & 0.00 \\
\hline 33 & landing foot splay $(\mathrm{mm})$ & 98.13 & 12.70 & $58,75^{*}$ & 46.45 & 89.88 & 21.86 & 93.88 & 23.91 & 101.29 & 10.01 & $79,75^{*}$ & 16.94 & 92.13 & 15.19 \\
\hline 34 & forelimb grip strength $(\mathrm{kg})$ & 3.64 & 0.96 & 2.59 & 1.80 & 4.55 & 0.83 & 3.67 & 0.66 & 4.07 & 0.59 & 4.26 & 0.86 & 3.46 & 0.49 \\
\hline 35 & hindlimb grip strength $(\mathrm{kg})$ & 1.27 & 0.30 & 0.88 & 0.79 & 1.06 & 0.30 & 1.15 & 0.22 & 1.10 & 0.31 & 1.38 & 0.29 & 1.23 & 0.24 \\
\hline 36 & grip strength of all limbs $(\mathrm{kg})$ & 8.04 & 1.35 & $5,09^{*}$ & 3.30 & 15.94 & 22.21 & 7.68 & 0.66 & 9.26 & 0.78 & 7.80 & 1.19 & 8.38 & 0.80 \\
\hline 37 & food receiving (\%) & 100.00 & 0.00 & $20,00 * * *$ & 0.00 & 100.00 & 0.00 & 100.00 & 0.00 & 100.00 & 0.00 & 100.00 & 0.00 & 100.00 & 0.00 \\
\hline 38 & body temperature $(\mathrm{oC})$ & 37.54 & 0.25 & $35,94 * * *$ & 1.00 & $36,97^{*}$ & 0.56 & $36,89^{*}$ & 0.52 & 37.42 & 0.20 & $36,66 * * *$ & 0.47 & $36,68^{* * *}$ & 0.47 \\
\hline 39 & vertical activity ( $\mathrm{No} / 10 \mathrm{~min})$. & 144.25 & 60.04 & $9,25^{* * *}$ & 14.03 & $47,63 * * *$ & 39.14 & 91.50 & 61.37 & 92.00 & 48.81 & $39,25 * * *$ & 39.97 & 122.38 & 104.75 \\
\hline 40 & horizontal activity ( $\mathrm{No} / 10 \mathrm{~min}$.) & 444.75 & 141.10 & $77,13^{* * * *}$ & 91.88 & $230,50 * * *$ & 100.72 & $285,13^{*}$ & 135.86 & 401.14 & 73.50 & $155,00^{* * *}$ & 97.85 & 389.13 & 271.06 \\
\hline 41 & total motor activity (No/10 min.) & 589.00 & 150.05 & $91,13^{* * *}$ & 103.93 & $278,25^{* * *}$ & 133.44 & $376,63^{*}$ & 180.68 & 493.14 & 117.27 & $194,25 * * *$ & 131.00 & 511.50 & 268.65 \\
\hline & & $n=\xi$ & & $\mathrm{n}=5$ & & $\mathrm{n}=8$ & & $\mathrm{n}=8$ & & $\mathrm{n}=7$ & & $\mathrm{n}=8$ & & $\mathrm{n}=8$ & \\
\hline
\end{tabular}


Tab. 3: The values of soman-induced neurotoxic markers $(\mathrm{x} \pm \mathrm{s})$ measured at $7 \mathrm{~d}$ following soman challenge by FOB (1-32 - scored values, No $33-41-$ values in absolute units). Statistical significance (comparison to control values) - see Tab. 2.

\begin{tabular}{|c|c|c|c|c|c|c|c|c|c|c|c|c|c|c|c|}
\hline & 24 hours & Cont1 & & Soma & & $\begin{array}{l}\text { Panpa } \\
\text { Some }\end{array}$ & & $\begin{array}{r}\text { Pyridostig } 1 \\
\text { Biperic } \\
+ \text { Som }\end{array}$ & $\begin{array}{l}\operatorname{mine}+ \\
\text { len } \\
\text { an }\end{array}$ & $\begin{array}{r}\text { Soman }+ \\
\text { HI- } 6\end{array}$ & & $\begin{array}{r}\text { Panpa } \\
\text { Soman } \\
\text { HI- } 6\end{array}$ & & $\begin{array}{r}\text { Pyridostig } \\
+ \text { Biperi } \\
+ \text { Somar } \\
+ \text { HI- }\end{array}$ & $\begin{array}{l}\text { mine } \\
\text { den } \\
+\mathrm{A} \\
6 \\
\end{array}$ \\
\hline No & Marker & $\overline{\mathrm{x}}$ & $\pm \mathrm{s}$ & $\overline{\mathrm{x}}$ & $\pm \mathrm{s}$ & $\overline{\mathrm{x}}$ & $\pm \mathrm{s}$ & $\overline{\mathrm{x}}$ & $\pm \mathrm{s}$ & $\overline{\mathrm{x}}$ & $\pm \mathrm{s}$ & $\overline{\mathrm{x}}$ & $\pm \mathrm{s}$ & $\overline{\mathrm{x}}$ & $\pm \mathrm{S}$ \\
\hline 1 & posture & 1.00 & 0.00 & $3,40^{* * * *}$ & 2.07 & 1.00 & 0.00 & 1.63 & 0.92 & 1.38 & 0.74 & 1.00 & 0.00 & 1.75 & 0.89 \\
\hline 2 & catch difficulty & 2.00 & 0.00 & $1,20 * * *$ & 0.45 & $3,00^{* * * *}$ & 0.00 & 2.00 & 0.00 & 2.13 & 0.83 & 2.00 & 0.00 & 1.75 & 0.46 \\
\hline 3 & ease of handling & 2.00 & 0.00 & $1,20^{* * *}$ & 0.45 & $3,00^{* * *}$ & 0.00 & 2.00 & 0.00 & 2.00 & 0.00 & 2.00 & 0.00 & 1.75 & 0.46 \\
\hline 4 & muscular tonus & 0.00 & 0.00 & 0.20 & 1.10 & 0.14 & 0.38 & -0.13 & 0.35 & 0.00 & 0.00 & 0.00 & 0.00 & -0.25 & 0.46 \\
\hline 5 & lacrimation & 0.25 & 0.46 & 1.40 & 1.52 & $1,00^{* * * *}$ & 0.00 & 0.38 & 0.52 & 0.25 & 0.46 & $0,86^{*}$ & 0.38 & 0.13 & 0.35 \\
\hline 6 & palpebral closure & 1.00 & 0.00 & 1.80 & 1.79 & 1.00 & 0.00 & 1.00 & 0.00 & 1.00 & 0.00 & 1.00 & 0.00 & 1.00 & 0.00 \\
\hline 7 & endo-exophthalmus & 0.00 & 0.00 & -0.20 & 0.45 & 0.00 & 0.00 & 0.00 & 0.00 & 0.00 & 0.00 & 0.14 & 0.38 & 0.00 & 0.00 \\
\hline 8 & fur abnormalities & 0.00 & 0.00 & 1.40 & 3.13 & 0.00 & 0.00 & 0.00 & 0.00 & 0.00 & 0.00 & 0.00 & 0.00 & 0.00 & 0.00 \\
\hline 9 & skin abnormalities & 0.00 & 0.00 & 0.60 & 0.89 & 0.63 & 1.19 & 0.00 & 0.00 & 0.25 & 0.71 & 0.50 & 0.93 & 0.00 & 0.00 \\
\hline 10 & salivation & 0.00 & 0.00 & $0,80^{*}$ & 0.84 & 1.00 & 0.00 & 0.00 & 0.00 & 0.13 & 0.35 & 0.00 & 0.00 & 0.00 & 0.00 \\
\hline 11 & nose secretion & 0.50 & 0.53 & 1.60 & 1.34 & $1,00^{*}$ & 0.00 & 0.63 & 1.06 & 0.88 & 0.35 & 0.86 & 0.38 & 0.50 & 0.53 \\
\hline 12 & rearing & 5.75 & 3.58 & 6.20 & 5.02 & 7.00 & 5.26 & $1,13^{* *}$ & 1.36 & 9.57 & 6.48 & 5.14 & 4.49 & 2.38 & 3.85 \\
\hline 13 & urination & 1.50 & 3.51 & 2.00 & 4.47 & 4.00 & 6.95 & 0.63 & 1.41 & 0.29 & 0.76 & 1.71 & 2.98 & 2.88 & 6.17 \\
\hline 14 & defecation & 1.25 & 2.05 & 0.00 & 0.00 & 1.57 & 1.40 & 1.00 & 1.20 & 0.63 & 1.19 & 1.86 & 1.21 & 0.63 & 0.92 \\
\hline 15 & clonic movements & 0.00 & 0.00 & $3,40^{* * * *}$ & 0.89 & $1,57^{*}$ & 1.51 & 1.13 & 1.55 & 0.25 & 0.71 & $1,29^{*}$ & 1.60 & 0.50 & 0.93 \\
\hline 16 & tremor & 0.00 & 0.00 & $2,40 * * *$ & 1.34 & $1,29^{*}$ & 1.60 & 1.00 & 1.41 & 0.43 & 1.13 & $1,29^{*}$ & 1.60 & 0.00 & 0.00 \\
\hline 17 & tonic movements & 0.00 & 0.00 & 0.80 & 1.30 & 0.00 & 0.00 & 0.00 & 0.00 & 0.00 & 0.00 & 0.00 & 0.00 & 0.00 & 0.00 \\
\hline 18 & gait & 0.00 & 0.00 & $2,20^{* * * *}$ & 2.17 & $1,86^{* * * *}$ & 0.38 & 0.13 & 0.35 & $0,50^{*}$ & 0.76 & $2,00 * * *$ & 0.00 & 0.00 & 0.00 \\
\hline 19 & ataxia & 0.00 & 0.00 & $1,40^{* * * *}$ & 0.55 & $0,71^{* * *}$ & 0.49 & 0.13 & 0.35 & $0,38^{*}$ & 0.52 & 0.00 & 0.00 & 0.00 & 0.00 \\
\hline 20 & gait score & 1.00 & 0.00 & $2,40^{* * * *}$ & 0.89 & $2,14^{* * * *}$ & 0.38 & 1.38 & 0.52 & $1,38^{*}$ & 0.52 & $2,00^{* * * *}$ & 0.00 & 1.25 & 0.46 \\
\hline 21 & mobility score & 1.00 & 0.00 & $2,00 * *$ & 0.71 & $1,71^{* * * *}$ & 0.49 & 1.00 & 0.00 & 1.00 & 0.00 & $1,57^{*}$ & 0.53 & 1.00 & 0.00 \\
\hline 22 & arousal & 3.63 & 0.74 & 4.00 & 1.41 & 3.71 & 0.95 & 2.88 & 0.99 & 4.00 & 1.20 & 3.86 & 0.69 & 3.25 & 0.89 \\
\hline 23 & tension & 0.00 & 0.00 & 0.40 & 0.89 & 0.00 & 0.00 & 0.00 & 0.00 & 0.00 & 0.00 & 0.00 & 0.00 & 0.00 & 0.00 \\
\hline 24 & stereotypy & 0.00 & 0.00 & 0.00 & 0.00 & 0.00 & 0.00 & 0.00 & 0.00 & 0.00 & 0.00 & 0.00 & 0.00 & 0.00 & 0.00 \\
\hline 25 & bizzare behavior & 0.00 & 0.00 & 0.00 & 0.00 & 0.00 & 0.00 & 0.00 & 0.00 & 0.00 & 0.00 & 0.00 & 0.00 & 0.00 & 0.00 \\
\hline 26 & approach response & 1.88 & 0.64 & 1.80 & 0.45 & 2.00 & 0.82 & 1.88 & 0.35 & 1.86 & 0.38 & 2.00 & 0.00 & 1.75 & 0.46 \\
\hline 27 & touch response & 1.88 & 0.35 & 1.60 & 0.55 & 1.86 & 0.38 & 1.88 & 0.35 & 1.57 & 0.53 & 1.71 & 0.49 & 2.25 & 0.46 \\
\hline 28 & click response & 2.00 & 0.00 & 2.00 & 0.00 & 2.14 & 0.38 & 2.00 & 0.00 & 2.29 & 0.76 & 2.00 & 0.00 & 2.00 & 0.00 \\
\hline 29 & tail-pinch response & 2.00 & 0.00 & 1.80 & 0.45 & 2.14 & 0.38 & 1.75 & 0.46 & 2.00 & 0.00 & 2.29 & 0.76 & 1.75 & 0.46 \\
\hline 30 & pupil size & 0.00 & 0.00 & -0.40 & 0.89 & 0.00 & 0.00 & 0.00 & 0.00 & 0.00 & 0.00 & 0.00 & 0.00 & 0.00 & 0.00 \\
\hline 31 & pupil response & 1.00 & 0.00 & 0.80 & 0.45 & 1.00 & 0.00 & 1.00 & 0.00 & 1.00 & 0.00 & 1.00 & 0.00 & 1.00 & 0.00 \\
\hline 32 & righting reflex & 1.00 & 0.00 & $2,20^{*}$ & 1.64 & 1.00 & 0.00 & 1.13 & 0.35 & 1.00 & 0.00 & 1.00 & 0.00 & 1.00 & 0.00 \\
\hline 33 & landing foot splay $(\mathrm{mm})$ & 97.19 & 12.28 & 67.60 & 38.97 & 93.64 & 18.69 & 99.38 & 22.18 & 107.29 & 23.11 & 90.07 & 20.82 & 100.00 & 22.94 \\
\hline 34 & forelimb grip strength $(\mathrm{kg})$ & 4.38 & 0.81 & 3.56 & 2.03 & 4.01 & 0.43 & 4.35 & 1.05 & 4.37 & 0.84 & 5.13 & 1.72 & 4.18 & 0.82 \\
\hline 35 & hindlimb grip strength $(\mathrm{kg})$ & 1.43 & 0.20 & 0.90 & 0.55 & 1.20 & 0.51 & 1.66 & 0.34 & 1.26 & 0.26 & 1.13 & 0.45 & 1.23 & 0.24 \\
\hline 36 & grip strength of all limbs $(\mathrm{kg})$ & 9.60 & 1.31 & 7.26 & 4.27 & 9.11 & 0.59 & 10.59 & 1.57 & 10.81 & 1.43 & 8.37 & 2.45 & 10.00 & 1.39 \\
\hline 37 & food receiving (\%) & 100.00 & 0.00 & $71,4^{*}$ & 48.80 & 100.00 & 0.00 & 100.00 & 0.00 & 100.00 & 0.00 & 100.00 & 0.00 & 100.00 & 0.00 \\
\hline 38 & body temperature $(\mathrm{oC})$ & 37.29 & 0.11 & 36.30 & 2.41 & 37.33 & 0.33 & 37.21 & 0.17 & 37.64 & 0.46 & 37.44 & 0.19 & 37.38 & 0.22 \\
\hline 39 & vertical activity ( $\mathrm{No} / 10 \mathrm{~min})$. & 95.38 & 25.83 & 129.75 & 74.72 & 50.14 & 41.64 & 84.38 & 73.06 & 78.86 & 9.01 & 85.29 & 42.13 & 100.00 & 49.23 \\
\hline 40 & horizontal activity ( $\mathrm{No} / 10 \mathrm{~min})$. & 342.75 & 57.76 & 417.50 & 107.24 & 259.43 & 110.30 & 303.50 & 191.38 & 349.57 & 29.74 & 362.57 & 43.21 & 358.00 & 72.56 \\
\hline 41 & total motor activity $(\mathrm{No} / 10 \mathrm{~min})$. & 438.13 & 77.48 & 312.71 & 317.87 & 309.57 & 148.21 & 387.88 & 258.47 & 428.43 & 36.34 & 447.86 & 79.22 & 458.00 & 118.82 \\
\hline & & $\mathrm{n}=\varepsilon$ & & $\mathrm{n}=5$ & & $\mathrm{n}=8$ & & $\mathrm{n}=8$ & & $\mathrm{n}=7$ & & $\mathrm{n}=8$ & & $\mathrm{n}=8$ & \\
\hline
\end{tabular}




\section{Results}

The results of the evaluation of soman-induced neurotoxicity at 24 hours and 7 days following soman poisoning are summarized in Table 2 and 3. Three non-treated somanpoisoned rats and one soman-poisoned rat treated with HI-6 and atropine died within 24 hours following soman administration. All pretreated soman-poisoned rats survived till the end of experiment ( 7 days following the intoxication).

The evaluation of soman-induced neurotoxic signs at 24 hours following intoxication proved significant alteration of 27 observed parameters. Soman caused relatively passive behaviour of rats during catching and handling. The animals were hypotonic. Soman also caused an increase in lacrimation, palpebral closure, nose secretion and skin as well as fur abnormalities. The exploratory activity in the open field was significantly decreased $(\mathrm{p}<0.05)$. Abnormal clonic movements appeared, gait and mobility were impaired $(p<0.05)$. Soman influenced response of animals to approach of the object and pupil response to light too. Grip strength of all limbs as well as the distance between hindpaws after a jump were significantly diminished $(p<0.05)$, animal's spontaneous horizontal as well as vertical motor activity, food receiving and body temperature were significantly $(\mathrm{p}<0.001)$ decreased (Table 2$)$.

When pharmacological pretreatment (PANPAL or pyridostigmine in combination with biperiden) or antidotal treatment (atropine in combination with HI-6) were administered alone, a few soman-induced neurotoxic signs were only eliminated. On the other hand, if pharmacological pretreatment was combined with antidotal treatment, the soman-poisoned rats were sufficiently protected from acute neurotoxicity of soman. Rats pretreated by pyridostigmine in combination with biperiden and treated by atropine in combination with HI-6 showed the best protection from soman-induced neurotoxicity compared to other groups at 24 hours following soman challenge because this combination was able to eliminate the majority of soman-induced signs of neurotoxicity (excluding abnormal clinic movements, reduced rearing, arousal and rectal body temperature) (Tab. 2).

Passive behaviour of rats during catching and handling, tremor, abnormal clinic movements, impaired gait and mobility score and abnormal righting reflex were only observed in soman-poisoned rats at 7 days following soman administration. While PANPAL pretreatment alone or in combination with antidotal treatment was not able to eliminate soman-induced signs of neurotoxicity, the combination of pyridostigmine with biperiden eliminated all soman-induced neurotoxic signs when it was combined with antidotal treatment (Tab. 3).

\section{Discussion}

In the case of a threat of soman exposure, it seems to be very important to have sufficiently effective pretreatment because soman-induced toxic effects are extraordinarily dif- ficult to counteract due to very low reactivating efficacy of currently used oximes $(6,7,12,19)$. Pyridostigmine that is stockpiled by various armed forces including the US army for pretreatment purpose against nerve agent poisoning is not sufficiently effective to increase the resistance of soman-exposed experimental animals (11) because it is only able to protect peripheral AChE from irreversible somaninduced AChE phosphonylation while soman can readily cross the blood-brain barrier and, therefore, exert its deleterious effects through its central toxic effects including centrally mediated seizures (2). The addition of centrally acting anticholinergic drugs to pyridostigmine for pharmacological pretreatment of acute soman exposures seems to be rational because a mixture of pyridostigmine with anticholinergic drugs should be able to increase the resistance of soman-poisoned animals and eliminate side effects of pyridostigmine, especially the effects of accumulated ACh (11).

The prophylactic efficacy of two various combinations of pyridostigmine with anticholinergic drugs were compared in this study. The combination of pyridostigmine with BNZ and THP stockpiled by Czech armed forces as PANPAL and Bulgarian prophylactic mixture consisting of pyridostigmine and biperiden. Both mixtures seem to be useful to increase the resistance of soman-poisoned rats and to increase the neuroprotective effects of common antidotal treatment of acute soman poisonings, nevertheless, the combination of pyridostigmine with biperiden appears to be more efficacious compared to PANPAL to eliminate acute soman-induced signs of neurotoxicity. Thus, biperiden that is characterized by high selectivity to $\mathrm{m}_{1}$ muscarinic acetycholine receptors in the central nervous system (9) was found to be more efficacious to eliminate soman-induced centrally mediated seizures than BNZ and THP involved in PANPAL. Therefore, it should be considered as means for the currently used pretreatment of the nerve agent poisoning, especially in the case of the threat of exposure to soman.

In conclusion, the combination of prophylactic antidotal mixture containing pyridostigmine and centrally active anticholinergic drugs with common antidotal treatment consisting of anticholinergic drug (mainly atropine) and oxime (mainly obidoxime, pralidoxime or HI-6) seems to be sufficiently effective to counteract acute neurotoxic effects of soman.

\section{Acknowledgement}

The authors express their appreciation to E. Reslová and J. Uhlírová for their skill technical assistance. The study was supported by the Linkage Grant SST.CLG.978.337. The authors appreciate the financial support from Scientific and Environmental Affairs Division in Brussels.

\section{References}

1. Anderson DR, Harris LW, Woodard CL, Lennox WI. The effect of pyridostigmine pretreatment on oxime efficacy against intoxication by soman or VX in rats. Drug Chem Toxicol 1992;15:285-94. 
2. Bajgar J. Present view on toxidynamics of soman poisoning. Acta Med (Hradec Králové) 1996;39(4):101-5.

3. Bajgar J, Fusek J, Vachek J. Treatment and prophylaxis against nerve agent poisoning. ASA Newslett 1994;94(4):10-7.

4. Dawson RM. Review of oximes available for treatment of nerve agent poisoning J Appl Toxicol 1994;14:317-31.

5. Frantík E, Hornychová M. Clustering of neurobehavioral measures of toxicity. Homeostasis 1995;36:19-25.

6. Kassa J. Comparison of efficacy of two oximes (HI-6 and obidoxime) in soma poisoning in rats. Toxicology 1995;101:167-74.

7. Kassa J. Review of oximes in the antidotal treatment of poisoning by organophosphorus nerve agents. J Toxicol - Clin Toxicol 2002;40(6):803-16.

8. Kassa J, Bajgar J. The influence of pharmacological pretreatment on efficacy of HI-6 oxime in combination with benactyzine in soman poisoning in rats. Hum Exp Toxicol 1996;15:383-8

9. Kassa J, Frantová K, Hoder P, Patočka J. A comparison of the efficacy of cholinolytics atropine and biperiden $\left(\right.$ Akineton $^{\mathrm{R}}$ ) in combination with HI-6 on cholinergic and stressogenic effects of soman in rats. Homeostasis 1996;37(3):135-6.

10. Kassa J, Fusek J. The positive influence of a cholinergic-anticholinergic pretreatment and antidotal treatment on rats poisoned with supralethal doses of soman. Toxicology 1998:128:1-7.

11. Kassa J, Vachek J, Bajgar J, Fusek J. A combination of pyridostigmine with anticholinergic drugs: effective pharmacological pretreatment of soman-poisoned mice. ASA Newslett 2001;84:16-9.

12. Lallement $\mathrm{G}$, Clarenson D, Brochier $\mathrm{G}$ et al. Eficacy of atropine/pralidoxime/diazepam or atropine/HI-6/prodiazepam in primates intoxicated by soman Pharmacol Biochem Behav 1997;56:325-32.

13. McLeod CG, Singer W, Harrington DG. Acute neuropathology in soman poisoned rats. Fundam Appl Toxicol 1984;5:53 8

14. Moser VC, Tilson HA, McPhail RC et al. The IPCS collaborative study on neurobehavioral screening methods: II. Protocol design and testing procedures. Neurotoxicology 1997a;18:929-38.

15. Moser VC, Becking GC, Cuomo V et al. The IPCS collaborative study on neuro- behavioral screening methods: III. Results of proficiency studies. Neurotoxicology 1997b;18: 939-46.

16. Moser VC, Becking GC, Cuomo V et al. The IPCS collaborative study on neurobehavioral screening methods: V. Results of chemical testing. Neurotoxicology 1997c;18:969-1056.

17. Petras JM. Soman neurotoxicity. Fundam Appl Toxicol 1983;1:73 83 .

18. Samnaliev I. Assessment of the drug combination containing biperiden and cholinesterase reactivators as a prophylaxis against soman induced poisoning. Voj Zdrav Listy 2002;71(2):86-92.

19. Shih T-M. Comparison of several oximes on reactivation of soman-induced blood, brain and tissue cholinesterase activity in rats. Arch Toxicol 1993;7:637-46.

20. Taylor P. Anticholinesterase agents. In: Hardman JG, Limbird LE, editors. The Pharmacological Basis of Therapeutics, $9^{\text {th }}$ ed. New York: McGraw Hill, 1996 p. 161-76.

21. Tonduli LS, Testylier G, Masqueliez C, Lallement G, Monmaur P. Effects of huperzine used as pre-treatment against soman-induced seizures. Neurotoxicology 2001;22(1):29-37.

22. Vachek J, Kassa J, Fusek J, Bajgar J. Present possibilities of treatment of organophosphate poisoning. Sbor Věd. Prací VLA JEP Hradec Králové 1993; 116:67-96.

23. Vayron P, Renard PY, Taran F et al. Toward antibody-catalyzed hydrolysis of organophosphorus poisons. Proc Natl Acad Sci USA. 2000;97(13):7058-63.

Submitted February 2003.

Accepted May 2003.

Doc. MUDr. Jiř́i Kassa, CSc., P.O. Box 35/T,

Purkyně Military Medical Academy, 50001 Hradec Králové, Czech Republic. e-mail: kassa@pmfhk.cz 